\title{
HiggsToFourLeptonsEV in the ATLAS EventView Analysis Framework
}

\author{
Th. Lagouri, F. Barreiro, and J. del Peso
}

\begin{abstract}
ATLAS is one of the four experiments at the Large Hadron Collider (LHC) at CERN. This experiment has been designed to study a large range of physics topics, including searches for previously unobserved phenomena such as the Higgs Boson and super-symmetry. The physics analysis package HiggsToFourLeptonsEV for the Standard Model (SM) Higgs to four leptons channel with ATLAS is presented. The physics goal is to investigate with the ATLAS detector, the SM Higgs boson discovery potential through its observation in the four-lepton (electron and muon) final state. HiggsToFourLeptonsEV is based on the official ATLAS software ATHENA and the EventView (EV) analysis framework. EventView is a highly flexible and modular analysis framework in ATHENA and it is one of several analysis schemes for ATLAS physics user analysis. At the core of the EventView is the representative "view" of an event, which defines the contents of event data suitable for event-level physics analysis. The HiggsToFourLeptonsEV package, presented in this paper, prepares the data for the given analysis context on the Analysis Object Data (AOD) files, the event-level physics analysis is performed and finally the output information is written as an Ntuple which can be read in stand-alone ROOT.
\end{abstract}

This paper describes the HiggsToFourLeptonsEV package and its structure as a collection of EVTools and EVModules. It also presents some illustrative results from the SM Higgs baseline

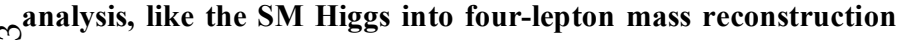
Ofor a nominal Higgs mass of $130 \mathrm{GeV}$. The lepton reconstruction óperformance as well as the SM Higgs to four leptons analysis Eperformance is studied in detail, in particular the dependence on Tkinematics, lepton reconstruction algorithms, isolation cuts and Heggs masses. Finally the paper discusses plans to adapt the code iinorder to produce Derived Physics Data (DPD) in POOL format Fityich can be read in ROOT or ATHENA, thus following the ARAS analysis model recommendations.

\section{INTRODUCTION}

he discovery of the Standard Model (SM) Higgs boson is the major goal of the ATLAS experiment at the Large 急然aron Collider (LHC). The experimentally cleanest signature for the discovery of the Higgs is its "golden" decay to four leptons (electrons and muons): $\mathrm{H} \rightarrow \mathrm{ZZ} \rightarrow 4 \ell$.

The excellent energy resolution of the reconstructed electrons and muons leads to a narrow 4-lepton invariant mass peak on top of a smooth background. The expected

Manuscript received November 14, 2008. This work was supported by the Spanish MICINN under contract FPA2005-03010.

Th. Lagouri is with the Physics Department of the Universidad Autonoma de Madrid (UAM), based at CERN, CH-1214, Geneva, Switzerland (telephone: 41227673007, e-mail: theodota.lagouri @cern.ch).

F. Barreiro, is with the Physics Department of the Universidad Autonoma de Madrid (UAM), Cantoblanco, 28049, Madrid, Spain. (e-mail: fernando.barreiro@uam.es).

J. del Peso is with the Physics Department of the Universidad Autonoma de Madrid (UAM), Cantoblanco, 28049, Madrid, Spain, (e-mail: jose.delpeso@uam.es). signal to background ratio after all experimental cuts depend on the Higgs mass itself. The major component of the background consists of irreducible $\mathrm{p} \mathrm{p} \rightarrow \mathrm{ZZ} \rightarrow 4 \ell$ decays. The most challenging mass region is between $120-150 \mathrm{GeV}$ where one of the $\mathrm{Z}$ bosons is "off shell" giving low transverse momentum leptons. In this region, backgrounds from $\mathrm{p} \mathrm{p} \rightarrow \mathrm{Zb} \overline{\mathrm{b}} \rightarrow 4 \ell$ and $\mathrm{p} \mathrm{p} \rightarrow \mathrm{t} \overline{\mathrm{t}} \rightarrow 4 \ell$ are important and require tight lepton isolation cuts to keep their contributions well below the $\mathrm{p} \mathrm{p} \rightarrow \mathrm{Z} \mathrm{Z}$ continuum.

HiggsToFourLeptonsEV is a physics analysis package based on the EventView (EV) [1] analysis framework within ATLAS ATHENA [2]. EventView is a suite of programs with a robust component model, which forms a general framework for ATLAS physics analysis in any context.

\section{II.HigGSTOFOURLEPTONSEV IN ATHENA EVENTVIEW AND EVENT DATA MODEL}

The development of the computing software required for the ATLAS experiment faced countless issues including full detector simulation, event reconstruction of the detector output, generation of Monte Carlo events and physics analysis.

To incorporate a wide variety of demands and to provide uniform interconnection among the offline software, the ATHENA framework was developed to assemble diverse subcomponents and external packages. Software projects, within the framework, share common interfaces and services, enabling them to communicate between each other.

Physics analysis is at the end of the computing workflow and it depends to a large extent on the rest of the framework. In-framework analysis is the place where one can obtain full accessibility to ATHENA reconstruction algorithms and, hence, general and powerful analysis tools can be developed within the ATHENA framework.

Several choices are offered to perform an analysis within ATHENA, where basically two different possibilities can be distinguished: with and without an additional analysis framework. Here, without an additional framework means that generic tools are plugged directly into ATHENA using the standard configuration via python scripts. Additional analysis code is typically added in form of tools and algorithms also callable directly from ATHENA. Here ATHENA acts directly as the analysis framework.

Running ATHENA with an additional analysis framework refers to cases where the used tools are either specific or wrapped generic tools and not plugged directly into ATHENA but into a specific framework which itself consists of one or 
more ATHENA algorithms. Such an additional analysis framework can be useful to organize common analysis tasks like bookkeeping, labeling of combinations and derived quantities. EventView is such an additional analysis framework [1].

For large collaborations like the ATLAS experiment, common interfaces and data objects are a necessity to insure easy maintenance and coherence of the software platform over a long period of time. In this respect the Event Data Model (EDM) plays the role of a common language to be utilized by the ATLAS software during its expected long lifetime. To enable physicists to analyze the data, additional stages of data sets are introduced [3], namely:

The Event Summary Data (ESD): which contains the detailed output of the detector reconstruction and will be produced from the raw data. It contains sufficient information to allow particle identification, track re-fitting, jet finding and calibration etc. thus allowing for the rapid tuning of reconstruction algorithms and calibrations. The target size for the ESD is $500 \mathrm{kB} /$ event.

The Analysis Object Data (AOD): which is a summary of the reconstructed event, and contains sufficient information for common analyses. The AOD can be produced from the ESD and the target size for the AOD is $100 \mathrm{kB} /$ event.

The Derived Physics Data (DPD): The $\mathrm{D}^{1} \mathrm{PD}$ that is obtained immediately from the AOD/(ESD) is referred to also as primary $D P D /($ Performance $D P D)$ and can be seen as a distilled version of the $\mathrm{AOD} /(\mathrm{ESD})$. A secondary $D P D$ $\left(\mathrm{D}^{2} \mathrm{PD}\right)$ is derived from primary DPD, retains the same format but is more specific to particular analyses. A tertiary DPD $\left(\mathrm{D}^{3} \mathrm{PD}\right)$ is derived from a primary or secondary DPD. It is used to produce the final plots used for publication and could be in another format, for example a small flat Ntuple or a collection of histograms.

Finally, there will also be "TAGs" on each event, indicating some general features of the event, and thus allowing quick access to them. The target size for the tags is $1 \mathrm{kB} / \mathrm{event}$.

A novel approach to physics analysis based on the "EventView" concept and an object oriented component model was developed. At the core of the "EventView" is the representative "view" of an event, which defines the contents of event data suitable for event-level physics analysis. EventView became a highly flexible and modular physics analysis framework in ATHENA.

The EventView analysis framework is a "sub-system" of ATHENA with a rich collection of generalized algorithms built around an analysis data object. It has proven its relevance to various physics working groups within ATLAS.

The Higgs ToFourLeptonsEV has a collection of specialized EventView tools useful for the Higgs to four-lepton analysis, which cover all of the event-level analysis. Typically, part of the event-level analysis is done within HiggsToFourLeptonsEV and the rest in ROOT [4].

The package HiggsToFourLeptonsEV is based on the common EVTools from the default EVToolkit and runs on Analysis Object Data (AOD) [3]. Truth, full reconstruction and trigger analysis are run in parallel and matching between them is performed after the insertion of objects into the corresponding view has been completed. At the same time, additional information of Final-State (FS) objects and matched objects are calculated in the analysis and stored in the User Data (UD) part of each EventView. This is written into separate ROOT TTrees using the Ntuple dumper. The HiggsToFourLeptonsEV analysis job can be sent to the computing Grid through the production and distributed analysis system PANDA [5], where all specified datasets are processed using the same analysis code.

In order to use Higgs ToFourLeptonsEV, one needs to check out the package and run it in the ATHENA framework. The user controls the standard HiggsToFourLeptonsEV operations through the main job options file.

\section{HiggstoFourleptonsEV IN THE ATLAS EVENTVIEW ANALYSIS FRAMEWORK AND ATLAS ANALYSIS MODEL}

The design of ATHENA [2] framework places emphasis on separation of data classes and algorithmic code. Its blackboard architecture style gives importance to the design of the data objects. Therefore, the Event Data Model (EDM) is an inherent part of the ATLAS computing model [6], which defines the analysis model of ATLAS.

The three main types of data needed in the analysis model are the AOD (Analysis Object Data), the TAG (small set of event-level meta-data), and the DPD (Derived Physics Data) having an average size of $10 \mathrm{kB} /$ event suitable for the final analysis.

The options for AOD based analyses or for the refined selection and DPD production step are:

1. AOD (direct) analysis: the AOD is accessed with ATHENA locally or on the grid, interactively or in batch mode and the output of the analysis in form of histograms is stored.

2. EventView based Ntuples: flat Ntuples produced with EventView and later analyzed with ROOT.

3. AthenaROOTAccess [3]: the AOD is accessed directly with ROOT and the transient ATHENA classes are used.

The existing ATLAS software infrastructure and the EDM forms the backbone of the EventView framework. Many of the components of the EventView framework are derived from the architecture of ATHENA. EventView fully benefits from ATHENA AlgTools and their configurability provided by Python to realize a flexible modular framework.

The EventView analysis framework within ATHENA allows the insertion, labeling, combination and association of EDM objects into a transient data store also called "EventView". Derived quantities from the inserted objects and combinations of them - called analysis data - can be added to the transient data store "EventView". Events are selected or rejected based on cuts on the inserted and combined objects and the derived data and a persistent version in the form of a ROOT TTree, can be saved to disk.

One key concept of EventView is the so called overlap removal, which allows the selection of a non-ambiguous set of 
input objects without double counting by retaining, in case of an overlap in $\eta-\varphi$, the already inserted object and rejecting the overlapping one. Other features are the bookkeeping capabilities in form of attachable labels to the inserted and derived objects. The tools acting on the labeled data are all configurable to handle labeled data accordingly.

The "EventView" is a collection of physics objects, which are coherent, exhaustive and mutually exclusive. "EventViews" are not unique; for each event a user may wish to consider the event with multiple different views.

The realization of the "EventView" EDM class consists of three types of sub-containers:

Final State (FS) Objects: Preselected objects considered in an analysis.

Inferred Objects (IO): Secondary objects reconstructed out of the final state objects.

UserData (UD): Variables calculated during the course of an analysis.

The "EventView" EDM class, the application manager EVToolLooper, and the EventViewBaseTool interface are the foundation of the EventView analysis framework.

The EVToolLooper being an ATHENA algorithm, manages the sequence of EVTools scheduled for analysis and the flow of the "EventView" object throughout the analysis.

The EVTool is an ATHENA AlgTool with EventView interface derived from EventViewBaseTool which implements the interface needed for algorithms written for EventView analyses, eg. calculator, associator tools.

The EVModule is a logical entity, which consists of one or more of the configured EVTools. It sets the variable parameters of generic EVTools and configures their behaviour within the analysis.

EVTools specific to the Higgs analysis context are developed within the HiggsToFourLeptonsEV package and specific configurations of general tools are stored in the form of EVModules. This includes object selection for the analysis, variable calculators and object reconstruction tools.

HiggsToFourLeptonsEV aims to be a common AOD analysis framework and its capability could range from copying AOD information into Derived Physics Data (DPD) to doing a complete analysis. A DPD is defined as a set of data, which is a subset of ESD or AOD content with the possible addition of analysis data (analysis data being defined as quantities derived from data in ESD or AOD). The role of the HiggsToFourLeptonsEV in the analysis model lies between the in-framework analysis using ATHENA and local (using DPD produced on Grid) analysis using ROOT. These are the two large functional components: writing and testing of analysis code using the HiggsToFourLeptonsEV (typically done on a CERN computing node or on a local machine with ATHENA installation), submission of the HiggsToFourLeptonsEV jobs to the Grid to produce DPD and further local processing using ROOT.

HiggsToFourLeptonsEV provides for the moment only flat Ntuples as DPDs (Tertiary $\mathrm{D}^{3} \mathrm{PD}$ ). However, there are plans to adapt the code in order to produce DPDs (Secondary $\mathrm{D}^{2} \mathrm{PD}$ ) in POOL format that can be read in ROOT with AthenaROOTAccess or in ATHENA.

\section{A. HiggsToFourLeptonsEV and ATLAS distributed analysis}

The ATLAS computing model is described in [6]. Raw data is reconstructed in the Tier 0 at CERN. The ESD, AOD and TAG are distributed to the Grid based computing system. The ten Tier 1 and their associated Tier 2 (and the CERN CAF) retain these data for subsequent processing. Each Tier 1 retains (approximately) $1 / 10$ of the raw data mostly on tape. The ESD, AOD and TAG residing at the Tier $1 / 2$ are the primary source for the start of ATLAS analysis. Two copies of the entire ESD data set are spread across the Tier 1 with one of these copies having a low latency for access (disk resident). Each Tier 1 receives a full copy of the AOD data, which is maintained in low latency storage in the Tier 1 and its associated Tier 2 cloud. Subsets of the ESD could also reside at Tier 2 to facilitate improved access. Each Tier 2 has a complete copy of the TAG data.

The Tier 1 provides CPU resources for re-reconstruction of raw data, calibration studies and user analysis. It is expected that the access to Tier 1 is restricted to production groups rather than individuals. The Tier 2 is responsible for simulation production (the output is retained at the associated Tier 1) and for user analysis: all members of the ATLAS virtual organization have access to Tier 2 resources. Some sites function as both a Tier 1 and a Tier 2 .

User, or group data such as DPD will reside at Tier 2. It is possible that some primary DPD could be resident at Tier 1 if this is needed to improve access. Tier 3 is a facility belonging to a single institute or group of institutes but they are not part of the common ATLAS resources.

Distributed analysis is a central part of any analysis in ATLAS. Considering the amount of data recorded by ATLAS (several PetaByte per year), it is impractical to try and process output data on a local resource. The Grid service PANDA provides a robust user front end for ATHENA job submission. Any ATHENA job can be sent with only very small modifications to the Grid resource and the result can be obtained through distributed data management (DDM) tools. Given these features of PANDA, HiggsToFourLeptonsEV needs very little extension to make the most of this service since it is already an ATHENA package. It does, though, have a small wrapper tool to help submission of HiggsToFourLeptonsEV jobs. 


\section{B. HiggsToFourLeptonsEV objectives and design}

The HiggsToFourLeptonsEV as shown in Fig. 1 is handling

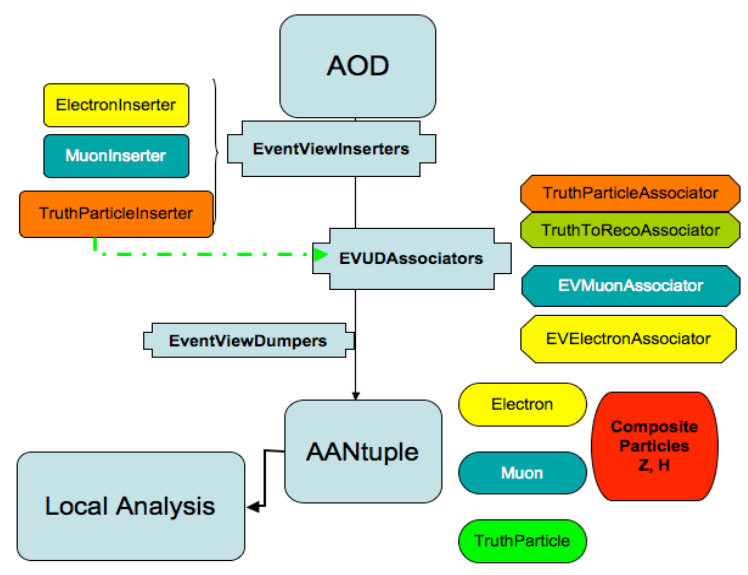

Fig. 1. HiggsToFourLeptonsEV general "flow" schema

Final State particles: "Muon" and "Electron" AOD objects, as inserted in the EV

Inferred objects: $\mathrm{Z}$ and $\mathrm{H}$ candidates as constructed from the final state particles (Muons, Electrons)

Truth Particles: "TrueMuon" and "TrueElectron" as inserted in the EV. "TrueZ" and "TrueH" are not inserted, but inferred from "TrueElectron/TrueMuon"

UserData: kinematic variables as calculated from the final state particles and the inferred particles (Z, H, "TrueZ/H") and written out to the Ntuple.

The HiggsToFourLeptonsEV package is used for performance studies like calculation of lepton reconstruction efficiencies and resolutions using the following EVTools:

EVUDToEVTruthParticleAssociators: reconstructed particles associated (matched) with the nearest truth particles (cut on their match in $\triangle \mathrm{R}$ ).

EVUDToEVMuonAssociators

and

EVUDToEVElectronAssociators: Truth Particles (TrueMuons, TrueElectrons) associated (matched) with the nearest (in $\Delta \mathrm{R}$ ) reconstructed particles.

Muon and electron resolution studies and fake muon, electron studies are done using the first tool and muon, electron resolution and efficiency studies are done using the second pair of tools.

The user controls standard HiggsToFourLeptonsEV operations through the main job options file. The flowdiagram is shown in Fig. 2.

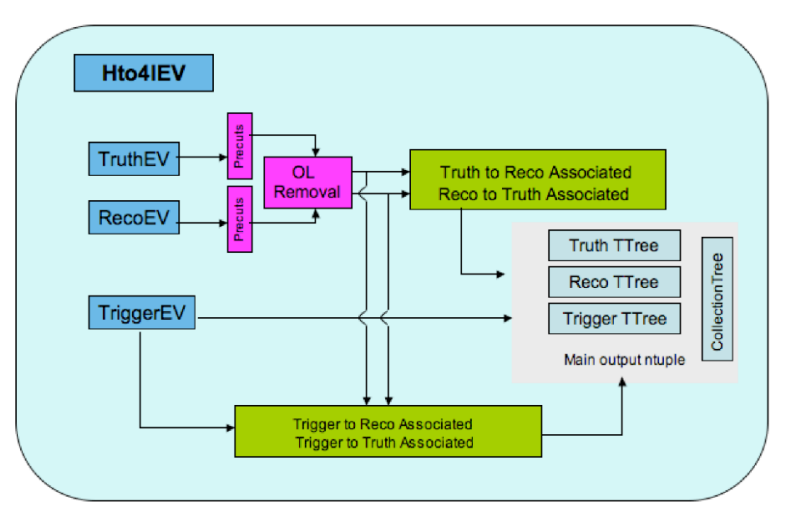

Fig. 2. Information flow of HiggsToFourLeptonsEV configuration job option (jobO).

The reconstructed objects, after the preselection cuts and overlap removals, are matched to truth objects (in $\Delta \mathrm{R} \leq 0.1$ ), after their preselection cuts and overlap removals with the "Reco to Truth Associated" python modules.

The truth objects, after preselection cuts and overlap removals, are matched to reconstructed objects (in $\Delta R \leq 0.1$ ), after their preselection cuts and overlap removals, with the "Truth to Reco Associated" python modules.

The trigger objects are matched with the truth and reconstructed objects (in $\Delta \mathrm{R} \leq 0.1$ ) after their preselection cuts and the overlap removals with the EVTrigger python modules.

The above associator modules, for example for the association of trigger objects to any kind of objects in EventView, loop over the EV objects with the specified labels we want to associate to a trigger object. Then, they associate the trigger object to the selected object with the EVUDToEVINav4MomAssociator EVTool.

The "TriggerView" inserts muons and electrons from all the trigger levels in the TriggerView and the properties of these muons and electrons are calculated in the UD for storing in the TriggerNtuple. The information on the "passed" trigger signatures from the "Trigger Decision" is also added in the Trigger Ntuple. The contents of the trigger view can be dumped to the screen for eventual debugging.

The reconstructed view, "RecoView", inserts the reconstructed muons and electrons with the EVTools, EVMuonInserter and EVElectronInserter respectively, after the preselection cuts and overlap removal cuts specific to the Higgs to four-leptons physics analysis. The combinatorial EVTools like "EVSimpleCombo" are used for Z mass (Zee, $\mathrm{Zmm})$ reconstruction and Higgs mass $(\mathrm{H} 4 \mathrm{e}, \mathrm{H} 4 \mathrm{~m}, \mathrm{H} 2 \mathrm{e} 2 \mathrm{~m}$, $\mathrm{H} 2 \mathrm{~m} 2 \mathrm{e})$ reconstruction. The kinematic properties of the inserted, final state (FS), reconstructed muons and electrons are calculated with the EVTools EVUDKinCalc, (EVUDMuonAll, EVUDElectronAll) and also those of the inferred objects (IO) Z, H. The contents of the "RecoView" can be dumped to the screen as well for debugging.

Finally, the "TruthView" is set-up by inserting true muons and electrons with the specific preselection cuts (the same as in the RecoView) and the overlap removal is again applied. The true combinatorics for $\mathrm{Z}$ and $\mathrm{H}$ combinations are 
scheduled as in the RecoView. The kinematic properties of the true FS objects electrons and muons and of the IO objects Z, H are calculated with the EVTools EVUDKinCalc, (EVUDTruthParticleAll).

In the next steps, the association of the trigger muon and electron objects to the true muon and electron objects and to the reconstructed muon and electron objects is being done using the associator modules mentioned above. Also, the reconstructed muons and electrons are associated to the true muons and electrons with their corresponding association modules.

After this, the ROOT TTree is created from the "TriggerView", "TruthView", and "RecoView" and the corresponding Ntuple files are saved.

\section{Object Insertion Order, Overlap Removal and Preselection Cuts}

The overlap removal priority is simply given by the order in which inserters are scheduled in a given view, so to change the priority one simply needs to change the order in which they are scheduled. The TriggerView has no overlap removal. The RecoView and TruthView by default perform checking for overlaps: if another object is already inserted within the defined cone of $\Delta R(\Delta R \leq 0.1)$, then the object will not be inserted. The insertion is done in EV in the following order: Muon, Electron, Photon, TauJet, ParticleJet.

The muon identification in ATLAS relies on the Muon Spectrometer (MS) for standalone reconstruction as well as on the Inner Detector (ID) and Calorimeters for combined muon reconstruction. In order to combine the muon tracks reconstructed in the ID and the MS, the ATLAS offline muon identification packages STACO [7, 8] and MUID $[9,8]$ have been developed. The purpose of these packages is to associate segments and tracks found in the MS with the corresponding ID track in order to identify muons at their production vertex with optimum parameter resolution.

In addition the MuTag [7, 8] and MuGirl [10,8] algorithms have been developed in order to recover muons, which fail to be reconstructed in the MS (either because they are low $\mathrm{p}_{\mathrm{T}}$ muons or because the number of muon stations is insufficient). The principle of these algorithms is based on the extrapolation of ID tracks to the inner stations of the MS and their matching to a segment reconstructed in these stations that was not yet associated to a combined track.

The details of electron reconstruction are described in [8]. In short, an electron is selected requiring:

1. A cluster in the barrel and endcap Liquid Argon (LAr) Electromagnetic Calorimeter (EMC), reconstructed by the ATLAS "egamma" offline software

2. An inner detector track associated with the cluster

3. Cluster containment in the LAr EMC

4. Consistency of the lateral shower shape of the cluster with an electron isolated from hadronic activity

5. The lateral shower shape to be inconsistent with a $\pi^{0} \rightarrow$ $\gamma \gamma$ decay, using the strip section of the LAr EMC
6. Track quality requirements: a certain number of hits on the pixel and silicon microstrip (SCT) trackers is required, and a transverse impact parameter smaller than $0.1 \mathrm{~cm}$.

The containment and isolation requirements $(2,3,4)$ are satisfied using the so-called "loose electron" definition. This definition uses shower shape variables calculated with the middle sampling of the LAr EMC. The addition of requirements 5 and 6 corresponds to the "medium electron" definition.

For this analysis, we use the muons with loose selection cuts, that means either good combined muons or low $\mathrm{p}_{\mathrm{T}}$ muons reconstructed with the following muon algorithms: STACO/MUID or MuTag/MuGirl respectively. For the electrons, we can use these reconstructed with loose or medium selection cuts.

A certain number of preselection cuts is applied to the (reconstructed, or truth) AOD objects. Any object failing such a preselection cut is not further considered, and is not written to the Ntuple at all. These preselection cuts mostly relate to the transverse momenta, quality of reconstruction or isolation criteria and are evolved through the needs and requests of the specific Higgs to four leptons analysis. Of course, for the true and reconstructed objects, an additional step is performed, namely the overlap removal.

\section{HigGSTOFOURLEPTONSEV BASELINE EXAMPLE ANALYSIS RESULTS}

The baseline example analysis of the Higgs to four leptons channel was performed with the HiggsToFourLeptonsEV analysis package [11], using the ATLAS Computing System Commissioning (CSC) offline release (13.0.40), consisting of a simple Higgs four lepton mass reconstruction, with $\mathrm{M}_{\mathrm{H}}=130$ $\mathrm{GeV}$.

HiggsToFourLeptonsEV selects in RecoEV and inserts 4 groups of leptons (two muons: Muon1, Muon2 and two electrons: Electron1, Electron2) in the event with specific reconstruction quality cuts from the corresponding AOD lepton container:

Electrons: reconstructed with medium selection cuts with the addition of calorimetric isolation using all cells (electromagnetic and hadronic) inside a $\Delta \mathrm{R} \leq 0.4$ cone are used (from "egamma" AOD container) [8, 11].

Muons: reconstructed with loose selection cuts (from "StacoMuonCollection" AOD container) are used $[8,11]$.

Inserted are all the leptons with removal overlap active, within $|\eta|<2.5$. The first "leading" group of leptons (Electron1/Muon1) is inserted with $\mathrm{p}_{\mathrm{T}}>20 \mathrm{GeV}$ and the second group of leptons (Electron2/Muon2) with $\mathrm{p}_{\mathrm{T}}>7 \mathrm{GeV}$.

Specific lepton isolation and impact parameter cuts are applied on the inserted leptons, in order to suppress the reducible backgrounds $(\mathrm{p} \mathrm{p} \rightarrow \mathrm{Z} \mathrm{b} \overline{\mathrm{b}} \rightarrow 4 \ell, \mathrm{p} \mathrm{p} \rightarrow \overline{\mathrm{t}} \overline{\mathrm{t}} \rightarrow 4 \ell$ ) below the irreducible-continuum one ( $\mathrm{p} \mathrm{p} \rightarrow \mathrm{Z} \mathrm{Z}$ continuum) $[8,11]$ :

Calorimeter Isolation: The sum of the transverse energy $\left(\mathrm{E}_{\mathrm{T}}\right)$ deposited in the calorimeter in a cone of radius $\Delta \mathrm{R}$ less 
than 0.2 around the electron or the muon $\left(\mathrm{SumE}_{\mathrm{T}}\right)$ is required to be less than $5 \mathrm{GeV}$.

Tracker Isolation: The sum of the transverse momenta $\left(\mathrm{p}_{\mathrm{T}}\right)$ of the inner detector tracks in a cone of radius $\Delta \mathrm{R}$ less than 0.2 around the electron or the muon track is required to be less than $5 \mathrm{GeV}$.

Impact Parameter (IP) Significance: The transverse impact parameter significance $(\mathrm{d} 0 / \sigma \mathrm{d} 0)$, where $\mathrm{d} 0$ is the measured perigee parameter of the track with respect to the reference point $(0,0)$, for electrons and muons is required to be less than 5.

The tracker isolation and the impact parameter significance variables are calculated separately with the HiggsToFourLeptonsEV EVUD calculator Tools: MuIsol and ElecIsol that inherit from the EVUDObjCalcBaseT EVTool and then these variables are stored in the UserData and into the HiggsToFourLeptonsEV Ntuple.

HiggsToFourLeptonsEV combines the two leading leptons and chooses the reconstructed $\mathrm{Z}$ mass combination: Zee1, $\mathrm{Zmm} 1$ that is within $\pm 15 \mathrm{GeV}$ around $\mathrm{M}_{\mathrm{Z}}=91.188 \mathrm{GeV}$. It also combines and calculates the mass of the two sub-leading leptons: Zee2, Zmm2 with $\mathrm{Z}^{*}$ mass $>20 \mathrm{GeV}$.

Finally, HiggsToFourLeptonsEV calculates invariant masses of the combined Higgs particles: H4e, H4m, H2e2m, $\mathrm{H} 2 \mathrm{~m} 2 \mathrm{e}$, with no Z-mass constraint, making the combinations of all the ZZ* available: Zee1Zee2, Zmm1Zmm2, Zee1Zmm2, Zmm1Zee2.

Similar steps are followed also in TruthEV for Truth Higgs mass calculation.

An example of this analysis is shown in Fig. 3 representing the reconstructed Higgs mass from all the lepton combinations fitted with a Gaussian.

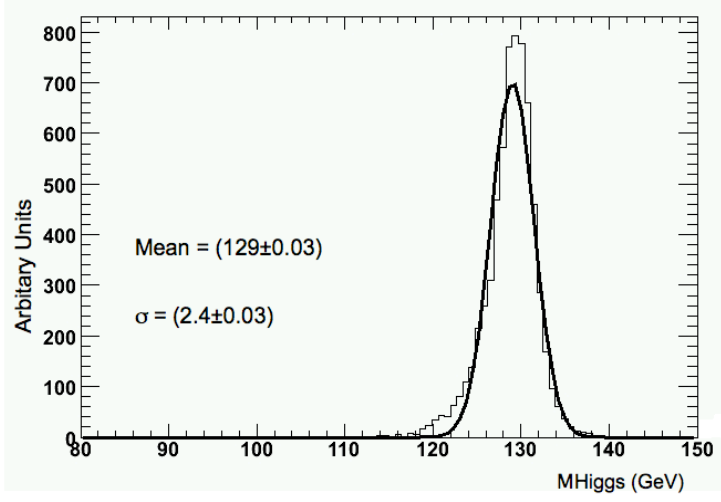

Fig. 3. Higgs mass as reconstructed with HiggsToFourLeptonsEV and fitted with a Gaussian.

\section{V.CONCLUSIONS AND FUTURE PLANS}

HiggsToFourLeptonsEV is a specific EventView physics analysis package for Higgs to four leptons analysis. It is developed as a collection of EVTools and EVModules, which are related to the Higgs to four leptons physics analysis context. Baseline example analysis results obtained with this package, using ATLAS CSC offline release (13.0.40) for DPD-flat Ntuple production were presented.

Future plans include the adaptation of the code, HiggsToFourLeptonsEV, for the new POOL format DPD production and analysis in ATHENA with EventView or in AthenaROOTAccess. Also, maintenance of the package is foreseen for the future ATLAS offline releases.

\section{ACKNOWLEDGMENT}

We would like to thank Kyle Cranmer, Amir Farbin and Akira Shibata and other EventView developers for their help. We would also like to thank Andreas Pfeiffer for useful discussions and Nicolas Kerschen for useful comments.

\section{REFERENCES}

[1] K. Cranmer, A. Farbin, A. Shibata, "EventView - the Design Behind an Analyis Framework", ATL-SOFT-PUB-2007-008, CERN, ATLAS Publication Note, 2007.

[2] ATLAS Collaboration, "ATHENA, The ATLAS Common Framework Developer Guide", ATLAS Internal, private comunication.

[3] D. Constanzo, I.Hinchliffe, S. Menke, "Analysis Model Forum Report", ATL-GEN-INT-2008-001, CERN, ATLAS Internal Note, 2008.

[4] Rene Brun and Fons Rademakers, "ROOT - an object oriented data analysis framework", Nucl. Inst. \& Meth. in Phys. Res. A 389 (1997), pp. 8186, 1997.

[5] T. Maeno, "PanDA: Distributed Production and Distributed Analysis System for ATLAS", Journal of Physics: Conference Series 119 (2008) 062036 (4pp), 2008.

[6] ATLAS Collaboration, "ATLAS Computing TDR", ATLAS TDR-017, CERN-LHCC-2005-022, 2005.

[7] S. Hassani et al., "A muon identification and combined reconstruction procedure for the ATLAS detector at the LHC using the (Muonboy, STACO, Mutag) reconstruction packages", 2007, Nucl.Instrum.Meth.A 572:77-79, 2007.

[8] ATLAS Collaboration, "Expected Performance of the ATLAS Experiment, Detector, Trigger and Physics", CERN-OPEN-2008-020, Geneva, 2008, to appear.

[9] Lagouri, Th. et al., "A muon identification and combined reconstruction procedure for the ATLAS detector at the LHC at CERN", Nuclear Science, IEEE Transactions on Volume 51, Issue 6, Dec. 2004 Page(s): 3030 - 3033

[10] Tarem, S., Panikashvili, N. "Low momentum muon identification in the ATLAS detector at the LHC", Nuclear Science, IEEE Transactions on Volume 53, Issue 3, June 2006 Page(s): 1304 - 1309

[11] Th. Lagouri, J. del Peso, "EventView Analysis Package Hto4lEV for Higgs to Four Leptons Searches", ATL-PHYS-INT-2008-028, CERN, ATLAS Internal Note, 2008 